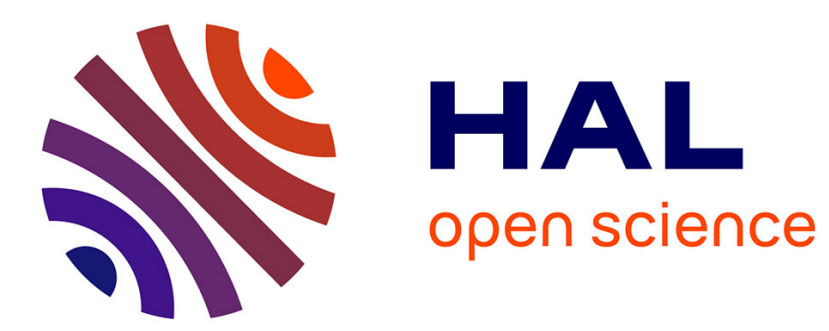

\title{
Du Texte Latin à la Concordance Imprimée
}

Philippe Fleury

\section{To cite this version:}

Philippe Fleury. Du Texte Latin à la Concordance Imprimée. Computers and the Humanities, 1986, Activities in France. Part II, 20 (4), pp.239-245. hal-03012950

\section{HAL Id: hal-03012950 https://hal.science/hal-03012950}

Submitted on 18 Nov 2020

HAL is a multi-disciplinary open access archive for the deposit and dissemination of scientific research documents, whether they are published or not. The documents may come from teaching and research institutions in France or abroad, or from public or private research centers.
L'archive ouverte pluridisciplinaire HAL, est destinée au dépôt et à la diffusion de documents scientifiques de niveau recherche, publiés ou non, émanant des établissements d'enseignement et de recherche français ou étrangers, des laboratoires publics ou privés. 

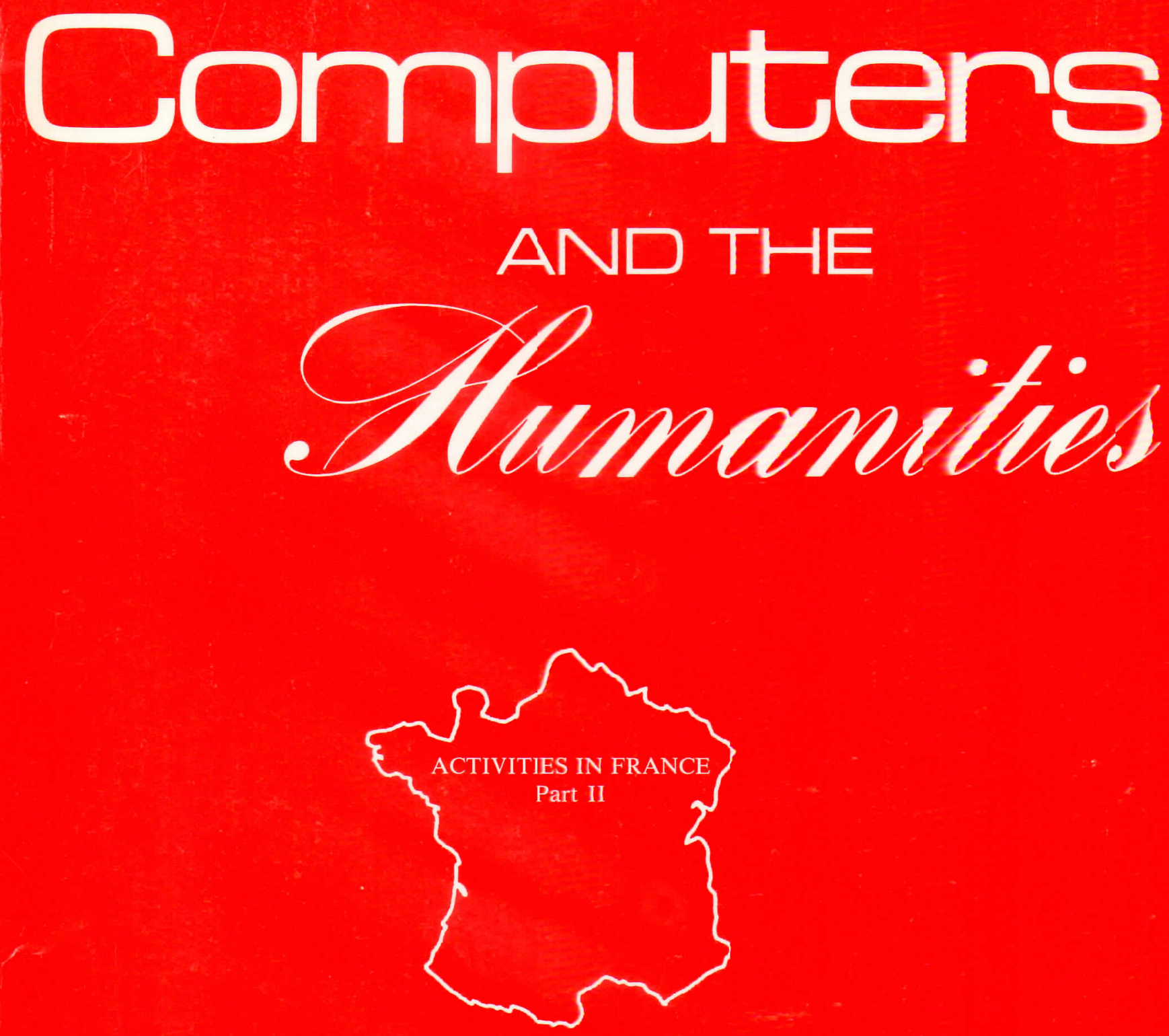

PARADIGM PRESS, INC.

OSPREY, FLORIDA 33559 


\title{
Du Texte Latin à la Concordance Imprimée
}

\author{
Philippe Fleury
}

Summary: The development of the technique of laser printing opens new ways for the writing of concordances to Latin texts, by means of electronic data processing. It is indeed possible to obtain as good a quality of printing as with traditional typography, even for a very important number of characters, which is always the case with concordances. A first step to obtaining perfection in the format of the final document consists in collecting data carefully and programming the software so as to provide scholars with a tool that will really facilitate their researches. Although the cutting out of the "useful context" is a very difficult problem to solve when using computers, as many data (punctuation, capital letters, diacritic signs... ) as possible can be collected from the very beginning. These data will then make the text provided for each keyword more comprehensible. The programming must obviously be improved to take all the collected data into account. The reader of a concordance produced in such a way will have at his disposal a text easy to read not only because of its typographic quality, but also because of all its information on reference, punctuation, lemma, manuscript tradition; also he will be able to find in appendices pertinent frequency lists, lists of names and all sorts of lexical, morphological, syntactical information according to the work involved in the data collecting and programming.

Lorsqu'en Europe, sous l'impulsion du L.A.S.L.A. ${ }^{2}$ de Liège, les techniques informatiques commencèrent d'être appliquées aux langues an-

Philippe Fleury is with the Centre d'Etudes et de Recherches pourl'Antiquité of the University of Caen. ${ }^{1}$ ciennes, le domaine de la concordence connut une certaine désaffection. Ce fut mêne l'objet d'un débat animé, dont la Revue des Etudes Latines ${ }^{3}$ s'est faite l'écho, entre Louis Delitte, directeur à l'époque du L.A.S.L.A. et "promoteur" des indices informatisés, $\epsilon t$ Pierre Grimal, déenseur des concordances traditionnelles et éditeır lui-même de concordances de Sénèque. ${ }^{4}$ En faitle fond du débat n'a jamais porté sur une opposition proprement dite entre index et concordance: la supériorité de la concordance, présentant les mots à l'intérieur de leur contexte, sur l'index qui ne donne que les références est théoriquement indériable. ${ }^{5}$ La question est de savoir s'il est possible et ntéressant d'informatiser le travail d'élabcration d'une concordance.

Une des premières concordances informatisées publiées sur un texte latin fut celle de Tite-Live par D.W. Packard. ${ }^{6}$ Diversement accueillie dans le monde des latinistes, on la recomut comme un document utile pour la recherche livienne, mais on lui reprocha l'absence de lemmatisation et surtout l'extrême confusion de la présentation. Devait-on pour autant condamner définitivenent toute perspective d'informatisation dans $\mathrm{e}$ domaine des concordances?

Depuis 1975j'ai travaillé sur cette question pour faire en sorte que les reproches faits jusqu'ici aux publications informatisées devienrent sans fondements. Mes efforts ont porté dans trois directions: - modernisation des moyens de saisie des textes et des possibilités de mise à jour des fichiers.

- affinage de la programmation pour prendre en compte le plus grand nombe possible de paramètres dans la constitution de la concordance (notamment en ce qui concerne la lemmatisation) 
-utilisation des techniques de l'impression laser pour offrir une présentation rivalisant avec l'imprimerie traditionnelle.

I. Saisie des Textes et Mise à Jour des Fichiers Il m'a parut nécessaire d'offrir au lecteur, dans le document final, une image absolument fidèle du texte de référence, en respectant non seulement la typographie (majuscules-minuscules ${ }^{7} .$. ) et la mise en page (pour les textes en vers notamment), mais aussi tous les signes de ponctuation simples (virgules, points-virgules, points. . .) ou complexes (parenthèses, guillemets... .) ainsi que les signes diacritiques. Le lecteur de notre concordance de Vitruve, par exemple, peut reconnaî tre aussi bien au niveau des formes, du contexte, que des lemmes, les mots qui posent un problème de tradition manuscrite, celui de la concordance de Cyprien peut distinguer immédiatement les mots ou les passages considérés par les éditeurs du Corpus Christianorum comme des citations bibliques.

Ce souci de fidélité a pour contre-partie une complexité plus grande de la saisie des textes et de la mise à jour des fichiers: il ne s'agit pas seulement d'enregistrer les uns à la suite des autres les mots d'un texte, il faut aussi tenir compte de tous les éléments qui nous permettront une restitution exacte de l'édition de référence. Pour faire face techniquement à ces problèmes, j'ai mis au point un système d'intercommunication entre les microordinateurs du C.E.R.L.A., ${ }^{8}$ l'ordinateur moyen du Centre de Calcul de l'Université de Caen ${ }^{9}$ et la puissante configuration du C.I.R.C.E. d'Orsay. ${ }^{10}$ Cette interconnexion me permet de concilier à la fois des impératifs d'économie (l'exploitation des micro-ordinateurs nécessite de faibles crédits de fonctionnement), de rapidité (la connexion en ligne directe avec le centre de calculde notre université nous donne immédiatement accès à de puissants logiciels à partir des micro-ordinateurs transformés en terminaux) et de puissance (le C.I.R.C.E. nous offre toutes les capacités de calcul et de stockage mémoire dont nous pouvons avoir besoin). En effet les fichiers que nous manipulons sont parfois assez volumineux: pour la concordance des Traités de Cyprien, par exemple, le fichier de départ représente 6.311 .760 caractères, le fichier d'impression 12.308.400. Il est donc plus intéressant pour nous d'utiliser, à la limite de leurs possibilités, d'abord nos micro-ordinateurs, ensuite l'ordinateur $\mathrm{d} \epsilon$ Caen et en dernier lieu le C.I.R.C.E. Nos données sont stockées au C.I.F.C.E. sur bandes magnétiques, mais la saisie et les nises à jour sont préparées surmicro-ordinateurs, avec un logiciel de traitement le texte. J'ai écrit ur programme de mise à jour en ?LI permettant d'effectuer en temps différé (batch) les corrections sur bandes magnétiques.

Il faut ajouter que l'utilisation di MINITEL 11 me permet, comme à n'importe quel chercheur, d'intervenir à tout moment et à partir dən'importe quel local muni d'une pris téléphonique, sur mes fichiers, soit pour les interroger, soit pour les traiter directement, sit enfin pour contrôler l'exécution des programmes. Cette innovation, à mon avis très importante pour nos disciplines, déruit l'argument de ceux qui envisageaient l'infornatique comme un instrument éservé à quelques privilégiés en raison du coût etde la rareté des appareils.

\section{La Programmation}

Une fois le texte saisi avec toutes les indications complémentaies dont je viens de parler, il s'agit de le traiter pour obtenir la concordance. J'aurais pu utiliser un prcgramme KWIC (KeyWord In Context), comme il en existe depuis longtemps dans beaucoup de Centres de Calcul. Ce programme aurait présentt toutes les formes da texte classées en ordre alphabétique avec un contexte paramétrable avant et après le mot,12 mais il m'a paru nécessaire d'aller plus loin et d'écrire moimême des prozrammes exactement adaptés aux textes latins etaux exigences de notre discipline.

Aller plus lin signifie lemmatiser les formes, adopter le système de référenciation usuel (qui n'est pas toujours le plus cohérent ni le plus logique informatiquement parlant...) pour l'auteur traité, délimiter le plus intelligemment possible le contexte, calculer lesfréquences en tenaut compte de la tradition manuscrite, maî triser totalement la mise en page.

\section{La lemmatisatisation}

L'intérêt de la lemmatisation n'est pas à démontrer, ${ }^{13}$ surtout pour une lansue telle que le latin. Il s'agit ctpendant d'un traval long et délicat pour le philolcgue auquel l'ordinateur n'apporte qu'une assistance. Nous utilisons pour cela le programme delemmatisation de Liège ${ }^{14}$ qui analyse automatizuement un grande nombre de 
formes et propose un choix de solutions pour les autres. Deux reproches ont été faits à la lemmatisation de Liège. Ils concernent d'une part le choix du dictionnaire de référence: le Lexicon Totius Latinitatis de Forcellini, ouvrage vieilli et peu utilisé aujourd'hui (il est pourtant le seul complet à ce jour), d'autre part l'utilisation d'indices numériques pour distinguer les homographes; il est évident que pour un lecteur qui ne dispose pas des codes d'analyse du L.A.S.L.A. la distinction entre ET 1 et ET 2 ou entre SVSPICIO 1 et SVSPICIO 2 n'est pas immédiatement déchiffrable.

Pour remédier à cela j'ai conçu un lexique de transformation des lemmes qui remplace certaines graphies du Forcellini15 (par exemple ADSVM au lieu de ASSVM, INQVAM au lieu de INQVIO . . .) et qui substitue aux indices numériques des indications plus claires (ET (adu.)" et "ET (coni.)" au lieu de "ET 1" et "ET 2"; "SVSPICIO, onis" et "SVSPICIO, ere" au lieu de "SVSPICIO 1" et "SVSPICIO 2"). Un programme PLI effectue la transformation automatiquement.

\section{La référenciation}

Chaque mot cité dans la concordance doit pouvoir être localisé très précisément dans le texte de référence. Or notre discipline connaî t divers types de référenciation suivant les auteurs et suivant les oeuvres: par livres, chapitres et paragraphes, par chapitres et lignes, par livres et vers etc. Certaines oeuvres comportent une préface, d'autres non. A l'intérieur d'une même oeuvre, un livre peut avoir une préface et le suivant non, etc. J'ai donc été amené à écrire un programme PLI tenant compte de tous ces paramètres et susceptible d'être modifié lorsque de nouveaux cas de figure se présenteront.16

Ainsi nos concordances ont une référenciation semblable à celle de nos dictionnaires usuels, avec la même précision qu'une référenciation transcrite "manuellement."

\section{La constitution du fichier concordance}

Un élément essentiel dans la constitution d'une concordance est le choix du "contexte utile" que l'auteur proposera pour accompagner chaque forme. Disons-le clairement tout de suite, jamais la machine ne pourra rivaliser avec le philologue dans ce domaine: d'un point de vue informatique, il s'agit donc de faire le mieux possible en proposant un contexte à la fois suffisammentlong pour être utile et sans mots superflus pour ne pas charger la présentation. C'est une question dificile, mais elle l'est aussi pour le philologue qui trataille manuellement. En effet ou bien le lecteur dela concordance sera intéressé par les associations ce mots et alors un contexte restreint lui sera suffisant, ou il s'attachera à la sémantique et dans ce cas, c'est parfois une page qu'il faudrait résumer four éclairer le sens d'un mot.

Les concordances de textes latins publiées à l'aide de moyens informatiques on: généralement recours à deux solutions simples: prur les textes en prose, le contexte est délimité par li longueur de la ligne d'impression (la forme étant placée au milieu), pour les textes de poésie, lecontexte choisi est souvent le vers. L'inconvénientde la première solution est d'offrir une présentation trop massive, encombrée de mots inutiles. La seconde solution est certes satisfaisante pour les nots placés en milieu de vers, mais elle conduit à ces aberrations pour les mots placés en début ou $\mathrm{n}$ fin de vers. ${ }^{17}$

Pour les textes en prose j'utilise ionc comme limites du contexte les signes choiss par l'éditeur lui-même pourmarquer les unités (e sens, c'est-àdire la ponctuation "forte" (./!/?//). Jamais je ne fais "descendre" ou "monter" le contexte après ou avant une ponctuation forte. Si cette solution ne permet pas de résoudre la question du contexte minimal, elle permet au moins d'obtenir une présentation plus aérée, sans mots assurément superflus. Je centre la forme au mileu de la ligne d'impression, puis je donne un conlexte à droite et à gauche, en luifixant pour limite naximale la longueur de la ligne d'impression ${ }^{18}$ cu la rencontre d'une ponctuation forte. Pour les tertes de poésie, j'ai adopté présentation vers par ver qui permet au lecteur d'avoir immédiatement comaissance de la métrique et de la place du mot dins le vers. La forme-clef est en majuscules afind'être repérée plus facilement. Lorsque cette forme est la première ou la deuxième du vers, je donne également le rers précédent; lorsqu'elle est la pénultième ou la dernière, je donnele vers suivant, cela toujours dans les limites de la rencontre d'une ponctuation forte.

\section{La documentation complémentaire}

Grâce au proģramme d'analyse cutomatique de C.I.P.L. de Liège, nous disposons dans notre fichier 
de départ d'informations concernant les catégories grammaticales, les genres, les cas, les voix, les modes, les temps. . . de chaque mot. Cela me permet d'éditer, à la suite de la concordance, plusieurs tableaux grammaticaux donnant la répartition des lemmes et des occurrences entre chaque catégorie grammaticale, et, à l'intérieur des catégories, entre les sous-catégories. Ces tableàux sort accompagnés de calculs de pourcentages.

La lemmatisation et l'enregistrement des signes diacritiques me permettent aussi de fournir des listes de fréquence pertinentes, éliminant par exemple du comptage les formes rejetées par l'éditeur (présentées cependant pour information à l'intérieur de la concordance avec un astérisque), distinguant le comptage des formes propres à l'auteur et celui des formes propres aux citations éventuellement. J'ai également écrit un programme pour comparer des listes de fréquence d'oeuvres ou d'auteurs différents et isoler ainsi des vocabulaires particuliers.

En marge de la documentation lexicographique et grammaticale, je me suis attaché aussi à informatiser la documentation bibliographique concernant chaque auteur traité. J'utilise à cet effet depuis 1984 le logiciel documentaire TEXTO ${ }^{19}$ qui offre toutes les facilités de création, de mises à jour, d'interrogation et d'impression. Pour Perse et Vitruve, nous avons imprimé ces bibliographies au début de la concordance, mais elles continuent d'être mises à jour.

\section{L'Impression Laser}

En impression classique (à impact), l'auteur d'un ouvrage volumineux se trouve toujours devant un dilemme: il faut choisir entre la rapidité (donc l'économie) et la qualité. 20

Pour la troisième de nos concordances, celle de Cyprien, j'ai utilisé la technique de l'impression au laser, qui, à mon avis, est en train de modifier actuellement de façon très sensible les perspectives de publication des travaux informatisés, en conciliant la rapidité des imprimantes classiques les plus performantes avec une qualité comparable à celle de l'impression traditionnelle.

Le principe de cette technique est le suivant: un faisceau laser se déplaçant dans les deux dimensions de la page ${ }^{21}$ est activé ou désactivé tous les 1/300 inch sur le matériel utilisé au C.I.R.C.E. (XEROX 9700). A chaque activation correspond un point noir sur la page. La capicité totale d'une page, exprinée en points, est tiéoriquement de $2478 \times 3507$ points. En termes de résolution on peut parler d $300 \times 300=90.000$ points par inch, ${ }^{2}$ ce qui donneune excellente qualté graphique. En fonctionnement standard (ligne à ligne), l'imprimante laser du C.I.R.C.E. a in débit nominal d'une page par seconde, quel que soit le taux de remplissagede cette page.

Le premier avantage de cette technique est donc l'association de la qualité et de la rapidité. Pour avoir un aperçu de la qualité obtenue, on consultera en Annexe une page de la concordance du Quod Idola, une ofuvre contestée de Cyprien, que nous avons éditéeà la suite de la concrrdance générale de ses traités. Cette concordanie générale comporte près de 80.000 occurrences; à raison de 83 lignes par page, elle occupe 1.118 pages et il a fallu à peine 19 minutes pour l'imprimer (soit plus de 4.800 lignesà la minute).

Le deuxième avantage, et non le moindre, est l'entière liberté laissée à l'auteur de choisir la forme et la taille des caractères, leur espacement ainsi que celui des lignes, la dimension des marges, l'orientationde la page, etc. Les perspectives ouvertes par ce type d'impression sont donc les mêmes que celles de l'imprimere classique. L'auteur peut mélanger divers types de caractères: romains et italiques par exemple, utiliser le gras et le souligné, imọimer les caractères grecs. . ., tout cela sans riei changer à la vitesse de l'impression.

A ce matériel de pointe est associé, au C.I.R.C.E., un système de traitement de texte, qui permet de rédiger intruductions, en-têtes, notes, tables des matières et de les imprimer cu laser avec les possibilités définies ci-dessus.

Pour peu cue l'on veuille s'en donner la peine, il paraî t donc possible aujourd'hui d'imprimer avec les moyens informatiques des concordances de qualité, au nême titre que celles produites "artisanalement," mais avec un gain de temps très appréciable et une grande sûreté. Certes le contexte est découpé moins "intelligemment" par la machine que par le philologue et le recours au texte s'avère parfois nécessaire, comme il s'avère aussi nécessaire (un peu moins souve.t il est vrai) dans les concordances "manuelles." Mail là nous rejoignons le débat concordance-index: la concordance est sûremert un instrument plus perfectionné, 
cependant elle occupe un volume environ trois fois plus important 22 que celui d'un index. Le choix de l'outil dépend finalement des objectifs de l'utilisateur; l'idéal étant de pouvoir disposer des deux pour chaque auteur. Néan moins, pour les deux types de travaux, il faut avoir les mêmes exigences de qualité pour un travail produit par les moyens informatiques que pour un livre classique. J'ai essayé de montrer que les techniques actuelles le permettent et si les précurseurs avaient quelques excuses dans ce domaine, on ne devrait plus pouvoir reconnaî tre aujourd'hui, en l'ouvrant en son milieu, un livre sorti des "presses de l'ordinateur."

\section{NOTES}

1. Dans le cadre du Centre d'Etudes et de Recherches pour l'Antiquité (anciennement C.E.R. de Langues Anciennes) de l'Université de Caen, un groupe de travail, dirigé par Louis Callebat, Professeur de Latin, s'applique depuis 1975, en collaboration avec le L.A.S.L.A. de Liège (cf. note infra), au traitement automatique des textes anciens. Les travaux entrepris tendent vers un double objectif:

1. Contribuer à fournir (pour les recherches lexicales, grammaticales, stylistiques) des instruments utiles d'analyse et d'interprétation: concordances, vocabulaires sélectifs, relevés grammaticaux, analyse des énoncés.

II. Constituer et exploiter une documentation spécialisée touchant un domaine particulier: celui des langages scientifiques et techniques latins.

Les oeuvres de Perse, Vitruve, Apulée (Apologie, Florides), Cyprien (Traités), Tertullien (De Pallio), Lactance, Dudon de St. Quentin, Guillaume de Jumièges, Guillaume de Poitiers sont ac-

tuellement en mémoire au C.E.R.L.A.

-P. Bout, L. Callebat, Ph. Fleury, M. Zuinghedau, Konkordanz zu den Satiren des Persius Flaccus, Hildesheim/New York, Olms, 1978. -id., Vitruve, De Architectura. Concordance, Hildesheim/Zürich New York, Olms/Weidmann, 1984

-P. Bouet, Ph. Fleury, A. Goulon, M. Zuinghedau (avec la collaboration de P. Dufraigne), Cyprien. Traités. Concordance, ibid., 1985.

2. On ne saurait trop insister sur le rôle de promotion joué par le Laboratoire d'Analyse statistique des Langues anciennes de Liège, au début des années 60 , dans le domaine de l'étude des langues anciennes par ordinateurs. Sur l'histoire du L.A.S.L.A., ses objectifs, ses méthodes, voir par exemple E. Evrard Le L. A.S. L. A.: "RELO" 1970,4 , p.1-16. C'est dans ce laboratoire, devenu depuis 1983 le C.I.P.L. (Centre Informatique de Philosophie et de Lettres) que je me suis initié en 1973 à l'application des méthodes informatiques au latin, et son actuel directeur, Joseph Denooz, a guidé avec beaurcoup de bienveillance et de competence mes premiers pas dans le d maine de la programmation en PLI. Je tiens ici à l'en remercier.

3. Cf. P. Grimal, Index et Concordances: "REL" XLIV, 1966, p.108-116.

4. L. Annaei Senecae Operum Moralium Concordantia, P. Grimal cura edita, Publications de la Faculté des Lettres et Sciences Humaines de Paris, Série "Textes et documents" VIII, Paris, PU.F. fasc.1 (Ad Marciam. De consolatione.) paru en 1965.

5. V. Lomanto (Concordantiae in Q. Aurelii Symmachi Opera, Hildesheim/New Jork, Olms/Weidmann, 1983,p.V.) oppose à tort concordances non lemmatisées et indices lemmatisés. En réalité, on peut très bien avoir des concordances lemmatisées et des indices non lemmatisés. La question de la lemmatisation est indépendante, à mon avis, du choix entre index et concordance (cf. infra). Mais son ouvrage est un exemple intéressant d'association des techniques informatiques avec un travail de finition classique permettant d'obtenir un outil remarquable au point de vue de la forme et du contenu. 6. D.W. Packard, A Concordance to Livy, Cambridge (Mass.), Har- vard Un. Pr. 1968 (4 vc.). A chaque mot de textecorrespond une ligne complète dans la concordance (une vingtine de mots en moyenne). Les formes ze sont pas lemmatisées $\epsilon$ les groupes de formes identiques ne sont pas isolés les uns des aures. IIn'y a donc pas de comptage de fríquence. L'absence de lenmatisation es justifiée par D.W. Packird dans son introduction lar l'importante intervention humaine cu'elle aurait demandée, e qui est exact: l'oeuvre complète de Tite Live représente tout di mème 505.000 mots et il a certainemertété plus utile aux spécialstes de Tite Live 作 devoir attendre des dizines d'annes un travail lenmatisé. Il faut reconnaî tre aussi que dpuis, des travaux non lemnatisés de qualité bien inférieure ont été publièes: la concordance diJ. Klecka (Concordantia in Publium Fipinium Statium, Hildeshim, Olms, 1983) par exemple allie conterte insuffisant, présentatic et typographie médiocres.

7. Conformément aux conventions de l'édition fraraise et de beaucoup d'éditions internationales, nous respectons limajuscule pour la première lettre des nots commencant une phrse. Ce détail est absent de beaucoup de tavaux informatisés. Un troail imprimé uniKonkordanz, Hildeshrim, Olms, 1979, est trè désagréable à consulter.

8. Un Zenith 100 et unZenith 150 compatible IEI PC.

9. MINI 6/43 BULL.

10. Un NAS9080 bprocesseur et un ADAHL470/V7. (C.I.R.C.E. $=$ Centre Intel Régional de Calciel Elecronique)

11. Certes ce terminal, listribué par les Postes et lélécommunications Françaises, offre un clavier et un écran peu cnfortables, mais des améliorations sont promises pour l'avenir. L'asence de traitement direct des caractres accentués, qui est adnissible pour les matériels conçus aux ISA, est tout de même un seu surprenante pour un matériel et unsystème de conception frncaise.

12. Cf. R. Busa, Concodantioe Senecae, Hildeskim, Olms, 1975 (2 vol.).

13. Il existe dans nos dsciplines un courant de pssée refusant la lemmatisation pour de raisons d'objectivité. Je le reprendrai pas icil'ensemble de ce délat qui est développé ailleus (voir par exemple "RELO" passim); e me contenterai simplenent de souligner trois points:

-il est vrai que la lemmatisation introduit unevertaine dose de subjectivité par les chox que l'auteur est amené lajire pour quelques regroupements (as par exemple des particpes-adjectifs en *to qu'un philologue ratachera à un lemme d'adjctif, qu'vn autre rattachera au lemme du verbe...). Mais lasubjectivité ne commence-t-elle pas dè le choix d'une édition de éférence et n'estce pas au fond la naturede tout travail de recherch d'étre Touvrage d'un auteur ou d'un goupe d'auteurs?

- par contre, je partag l'opinion de ceux qui disnt que, pour une oeuvre volumineuse, ilvaut mieux disposer rapiement d'un travail non lemmatisé qued attendre des années le reultat d' une lemmatisation. Ceci étant je ne pense pas que l'on pisse considérer un index ou une concor ance non lemmatisé come une fin en soi, mais comme une étapt vers un travail plus élabré

-l'idéal serait que les ravaux non lemmatisés scent simplement enregistrés sur support magnétiques et consultales par tout chercheur qui en ferait la dimande. Cette idée de barues de données consultables "à la cart" a été exprimée il y a dé longtemps, par E. Evrard notamment qui la rappelle à la fin d'in compte-rendu récent (Trois concordinces récentes d'auteurs bitins: "RELO" 1983, p.225).

14. Cf. J. Denooz, Recierches sur le traitement atonnatique de la langue latine: "RELC" 1973, 1.

15. Notre base de réféenceen matière de graphe des lemmes est l'Oxford Latin Dictinan fasc.1-8, Oxford, larendon Press, 1968-82. Les lemmesnon cités dans ce dictioneire sont choisis, dans tous les cas possibles, en reférence au Thawnus Linguae Latinae.

16. Lors de la saisie dutexte, des codes sont enrejistrés qui permettent de repérer: les fin: de chapitre, de paragraple de ligne etc. Le programme lui-même st paramétré pour tenir conpte des préfaces, des lignes ou des parasraphes qui ne commencot pas au numéro 1 , etc.

17. Admettons par exmple que le mot vedettesoit le premier du vers et qu'il soit suivi d'une ponctuation forte le seul contexte 
intéressant sera le vers précédent. Cf. Vir. Aen.2,13: Incipiam. Fracti bello fatisque repulsi

Seul le contexte de 2,12 est utile pour interpréter incipiam: Quamquam animus meminisse horrit luctuque refugit,

18. Je me limite à une seule ligne par formepour des raisons de volume global de l'ouvrage, mais aussi pour des questions de clarté. Il est à noter que ce principe n'est pas propre aux concordances informatisées: cf. H. Quellet, Concordanceverbale du DE CORO NA de Tertullien, Hildesheim/New York, Olms, 1975. La longueur de la ligne est paramétrée dans le progranme PLI de constitution de la concordance et elle peut être fixée selon le format choisi. Pour la concordance de Vitruve, j'ai utilisé des lignes de 118 caractères: 12 caractères pour la référenciation, 106 pour le contexte, ce qui représente en moyenne une quinzaine de nots.

19. Le logiciel TEXTO est le fruit de la collaboration d'une équipe de recherche de CHEMDATA (Lyon) avec le laboratoire du G.I.S (Groupement d'Intérêt Scientifique) de la Maison de l'Orient (C.N.R.S.) à Lyon
20. Il est vrai que des rogrès ont été réalisés par l'impression ra. pide classique: nos co cordances de Perse et de ritruve ont été sor. ties (en double impresion) sur les imprimantes apides du L.A.S.L.t et elles offrent, je cris, une typographie acceptable. Cependant, en poussant le souci e la qualitè à l' extrême, lameilleure impres sion à impact s'obtint aujourd'hui avec les mprimantes dites "oualité courrier" mis elles n'impriment pas pls de 20 caractères à la seconde: à ce rythe il nous aurait fallu près $(e) 3000$ heures pour à la seconde: à ce rythe il nous aurait fallu près ce 3000 heures

imprimer une conconlance de la taille de cellede vitruve! $\mathrm{cm}(8,26 \times 11,69$ incles $)$.

22. L'index comportée même nombre de lignesthéoriques qu'une concordance (une por chaque forme, plus une pour chaque lemme dans le cas de travur lemmatisés), mais lles peyvent être présentées sur trois olonnes. 


\section{ANNEXE}

$\begin{array}{lr}A(A B) \\ \\ 20 & 4 \\ 20 & 15 \\ 20 & 18 \\ 21 & 10 \\ 23 & 13 \\ 24 & 16 \\ 25 & 1 \\ 29 & 10 \\ 30 & 11 \\ 31 & 3 \\ 31 & 7\end{array}$

11

uisitur et sejulcrum elus ostenditur, et $A B$ eo Saturnum fugatum manifestum es: : inde uaria deorum religio mutatur, dum non unus $A B$ omnibus Deus colitur, sed propriaculdue potestat is proditum $s 1 b 1$ de dils hominibus a sacerdote serretum, quod malorum $\epsilon$ regum Mox A nescio quo FeDris dedicata et Acca et et a utgore caele:t terreno contagio

posteaquam terrenis $u 1$ ti is inmersi sunt Deo homines auocare et ad superstitionem sui $A B$ intellectu lerae religionis auertere, cum Deo homino spiritum sponte dimisit et die tertio rursus a mortuis sponie surrexit.

monominem, quem dilexit, quem indult quem A morte protex't, ad Patrem ulctor monente diffusi praecepta in salutem dare. AB errore tenetrarum ad ulam lucts educere,

AC, c. coni.; \& UId.ATQVE 3

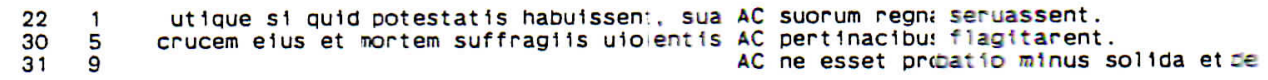

$21 \quad 11$

Mox a nescio Febris dedicata et ACCA'et Flora veretrices.

ACCENDO 1

302 et doctrina llle et sapient la reuincebat, ACCENSA 1ra et indignat fone prouocat, ut ACCIDO, (cado) 1

225 Regna autem non nerfto ACCIDVNT, sed sorte varfantur.

ACCIPIO 4

203 nec mercedem operis infelix instiuctor ACCEPIT.

2055 est: inde Lat lum de latebra elus nomen ACCEPIT. 233 aut augurils Romana regna creuerun:, sed ACCEPTVM tempu: certo 4 ine custodiur. de diuinis muneribus hauriren: quam ACCEPTAM I udae contempt is religionbus

AD $\quad 22$

$\begin{array}{rr}19 & 4 \\ 20 & 17 \\ 22 & 8 \\ 22 & 11 \\ 22 & 13 \\ 23 & 16 \\ 24 & 1 \\ 24 & 13 \\ 25 & 1 \\ 25 & 2 \\ 25 & 3 \\ 25 & 16 \\ 28 & 11 \\ 30 & 8 \\ 30 & 14 \\ 31 & 1 \\ 31 & 3 \\ 31 & 4 \\ 31 & 4 \\ 31 & 7 \\ 31 & 8 \\ 31 & 12\end{array}$

Inde 111 is inst 1tuta templa inde AD defunctorum uultus per imaginem ita esse Alexanjer magnus insigni uol umine AD matrem suam scripsit. metu sua Ceterum $S 1$ AD originem reieas, erubescas.

nunc ut rex ipse principatum nabeat $A D$ crimina, f1. Romulus parricida, coue ut nunc ut rex lose principatum fallunt $A D$ coplam ciulatis augendam: nupt ia sunt norunt et Socrates instrul se e: regi AD arbitrium diemonit praedicabat, ot magis daemonil praedicabat, et magis inde est AD perniciosa lel ludicra potentatu: quorum ual 1 tudinem frangunt. morbos lacessunt, Ut $A D$ cultum sul cogant. ut nidore alta iumet studium est quam a Deo nomines auocare et $A D$ superst itiolem sut ab intellectuceras cum sint ipsi poenales, quaerere sibi AO Doenam comies, quos ad Crimen sum. AD crimen suum fecerint a duorum hominem induit, quem pe-oucat $A D$ Patrem. mortem sed ut uinceret, et cum passus esset. AD superos denio regred1. Ut u1m divinae. mortem sed ut Uinceret, firmitate consicuus AD dies quadraginta remoratus est, it de po ad dies quadraginta remoratus est, ut de eO AD praecepta uitalía instrul possent et diex1t, quem induit, quem a morte protexit. AD Patrem ulctir inponeret, lam venurus e dilexit, quem induit, quem a morte protexit. AD patram diabil inpot ad censuram geeris ulctor inponeret. lam venturus e caelo ad poenam diaboli et $A D$ censuram geler is numani ultor is igore et

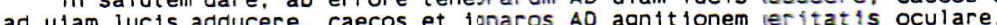

ut Christus Def Filius, qui nominious $A D$ uitam datus creditur, non tantum

ADDVCO

317 dare, ab errore tenebrarum ad ulam lucis ADDVCERE, caecis et ignaros ad agni:fonem ADFINIS

$26 \quad 3$

Pompelus et zaesar ADFINES fuerun nec tamen necessitulinis

ADFLATVS

248 adque imaginibus consecratis delitescunt, hi ADFLATV suo uaim pectora inspirant extorum 